\title{
Stem Cells and Tissue Engineering: An Overview of Modern Advances and Discoveries related to Stem Cells that contribute to Replacement of Native Tissue
}

\author{
Hope Johnson ${ }^{1}$ and Vincent Gallicchio ${ }^{2 *}$ \\ ${ }^{\prime}$ Department of Bioengineering, College of Engineering, Computing and Applied Sciences, Clemson University, South Carolina, USA \\ ${ }^{2}$ Department of Biological Sciences, College of Science, Clemson University, South Carolina, USA
}

\section{Article Info}

*Corresponding author:
Vincent Gallicchio
Department of Biological Sciences
122 Long Hall
College of Science
Clemson University
Clemson, South Carolina
USA
E-mail: vsgall@clemson.edu

Received: September 15, 2017

Accepted: October 16, 2017

Published: October 23, 2017

Citation: Johnson H, Gallicchio VS. Stem Cells and Tissue Engineering: An Overview of Modern Advances and Discoveries related to Stem Cells that contribute to Replacement of Native Tissue. Madridge J Clin Res. 2017; 1(1): $20-26$

doi: $10.18689 /$ mjcr-1000104

Copyright: ๑ 2017 The Author(s). This work is licensed under a Creative Commons Attribution 4.0 International License, which permits unrestricted use, distribution, and reproduction in any medium, provided the original work is properly cited.

Published by Madridge Publishers

\begin{abstract}
The number of patients that need engineered tissue replacements is on the rise. Tissue engineering is a subset of the field of regenerative medicine that patients can and should use over modern symptomatic treatments. Tissue-engineered constructs ensure patients receive the best care they possibly can by giving patients personalized care, intended specifically for them. Engineers use the patient's own stem cells to repair or replace irreparably diseased/damaged tissues. Although it is a comparatively new field of medicine, tissue engineering shows incredible promise largely thanks to the incorporation of stem cells.
\end{abstract}

Keywords: Stem cells; Tissue and Organ engineering.

\section{Introduction}

The current solution for patients with diseased tissues and/or organs is replacement, whether the replacement is prosthetic or allogeneic in nature. Sadly, these solutions are not failsafe. Prosthetic implants come with their own myriad of potential (and likely) problems. Even after intensive surgeries and long rehabilitation periods, it is possible for prosthetics to dislodge, become infected, fracture, and/or occlude [1]. These problems are often innocent ones associated with regular use of a foreign material in a damaged host. For example, a total hip replacement that sustains a high force (like in the case of a car accident) may fracture or dislodge despite a patient or surgeon's best efforts. On top of these unforeseeable or "innocent" problems, additional problems exist associated with surgeon malpractice or even recalls for implantable devices. A surgeon or medical device producer may knowingly or unknowingly incorporate materials incompatible with the patient or intended use. Reconstructive or replacement surgery is necessary should any of these problems occur. However, because surgery places such a huge strain on the patient's body, reconstruction puts an already unhealthy patient at risk for further health deterioration or even fatality.

Despite these issues, prosthetic implantation remains a more common option than organ transplantation. Many diseases, injuries, or even birth defects may necessitate an organ transplant. Organ transplantation, while a terrific concept, has three very specific issues associated with implantation and lifetime use. First of all, the demand for organs far exceeds the supply that is available for patient use. According to the U. S. Department of Health and Human Services, there are currently over 116,000 patients on the national transplant waiting list [2]. Every 10 minutes another person is added to the national transplant waiting list and every day 20 people die waiting for a transplant [2]. It is not enough, though, for organs to suddenly become available. Patients are screened to ensure that any organs 
they receive will not experience acute rejection. Even if patients are lucky enough to be matched with an organ, the patient will need a lifetime of immunosuppression drugs to prevent rejection.

Obviously, the current solution of replacing damaged or diseased tissues with either prosthetic or allogeneic implants is problematic at best. It is for this reason that the field of tissue engineering emerged in the mid-1980s [1]. The goal of which remains to create living replacements that, once implanted, become part of the body. Tissue engineering is an obvious solution to the problems of prosthetic failure and organ rejection. An organ engineered for the patient with the patient's own cells would not have the infection risks associated with a prosthetic or the adverse immune responses guaranteed by a transplant. In order to achieve full integration with the host, it is clear that host stem cells must be used to develop or generate functional tissue. The remainder of this paper will explore tissue-engineered products and the advances made towards their development. The paper will discuss the modern advances made in organ/tissue growth and summarize how current tools contribute to the creation and study of tissue-engineered products made for patient treatment. Additionally, the paper will touch on current research made by tissue engineers to direct stem cell regeneration without the need of a scaffold-tissue construct.

\section{Discussion}

There are multiple applications for tissue engineering in addition to the fabrication of organs or tissues that are approved by the U.S. Food and Drug Administration (FDA). Skin grafts are one such example of tissue engineering. Skin grafts may not necessarily contain cells but may contain an acellular scaffold or thin extracellular matrix-based material that contains various growth factors to promote recruitment of stem cells to the site for wound healing. A more specific example includes the autologous cellular product $\mathrm{MACl}$, which is used in knee joint cartilage repair [3]. Healthy cartilage is isolated from the joint that has experienced acute or repetitive trauma via biopsy and autologous chondrocytes are thereby extracted and expanded. The chondrocytes are loaded onto a collagen membrane and the product is then implanted to the defect area of the knee joint to relieve pain and restore natural function [3]. A final example, that I would argue epitomizes the pursuit of tissue engineering, is the production of a tissue-engineered trachea graft by seeding an acellular xenogeneic scaffold with autologous stem cells to replicate a host's trachea [19]. While seemingly different, all of these products have in common the need for a scaffold to stimulate cell growth.

Most tissue engineering techniques attempt to use or attract stem cells and direct their growth for an intended application. Researchers have found that scaffolds, oftentimes three-dimensional templates that mimic (or contain) extracellular matrix $(E C M)$, are essential for cellular adhesion and proliferation necessary for controlled growth provided that a few key requirements are met [5]. Scaffolds must provide mechanical support for stem cells and also have high pore interconnectivity [5]. Studies have shown that stem cells will only occupy a specific environment or niche in the body that scaffolds strive to emulate. So, it is essential for scaffolds to mimic a stem cell niche and also to allow space for future vascularization once cell growth has progressed far enough for the cells to become a tissue. It is also necessary for the scaffold to be biocompatible and biodegradable [5]. Once the cell development has progressed far enough, the scaffold should degrade safely in the host to allow for new tissue seeding and formation.

Stem cells are a clear choice for developing tissue due to their capability for differentiation. While it is possible to acquire adult host cells of the desired tissue, oftentimes these tissues are already diseased or degraded, thus the need for replacement. The most popular option for tissue engineering is pluripotent stem cells acquired from the inner cell mass (ICM) of an embryo. These stem cells will form into the three primary germ layers, which, as a whole, can then differentiate into all tissues of the body. Stem cells at this stage are induced into organization or specialization by coordinating their behavior with neighboring cells. Inductive signaling and other signaling pathways, like Notch or Hedgehog, are largely dependent on location of the cells and their proximity to the signals that are sent out. To engineer tissue growth properly, engineers construct scaffolds to stimulate and direct stem cell development.

Scaffolds are critical to inducing the desired formation of tissues with autologous stem cells. So, it is critical that scaffolds are made as specifically as possible. Popular fabrication techniques include solid free-form fabrication rapid prototyping, inkjet-printing system, and laser sintering [4]. These techniques ensure that complex geometries are fabricated on a micron scale with computer-aided design (CAD) models and then 3D-printed (Fig. 1). 3D printing allows tissue engineers to develop scaffolds to create patient- specific organs or tissues. The use of decellularized porcine or human organs as scaffolds for human cells is another scaffolding technique that is gaining considerable traction [4]. The organ is harvested, then subjected to a decellularization technique specific to the organ [4]. A common technique requires bathing the organ in a gentle decell solution, then agitating the organ in solution [4]. Following agitation in the decell bath, the organ will retain the natural ECM scaffold and the scaffold mechanical properties while losing all genetic material [4]. At this point, the scaffold is then seeded with the patient's own cells and incubated for recellularization (Fig. 1).

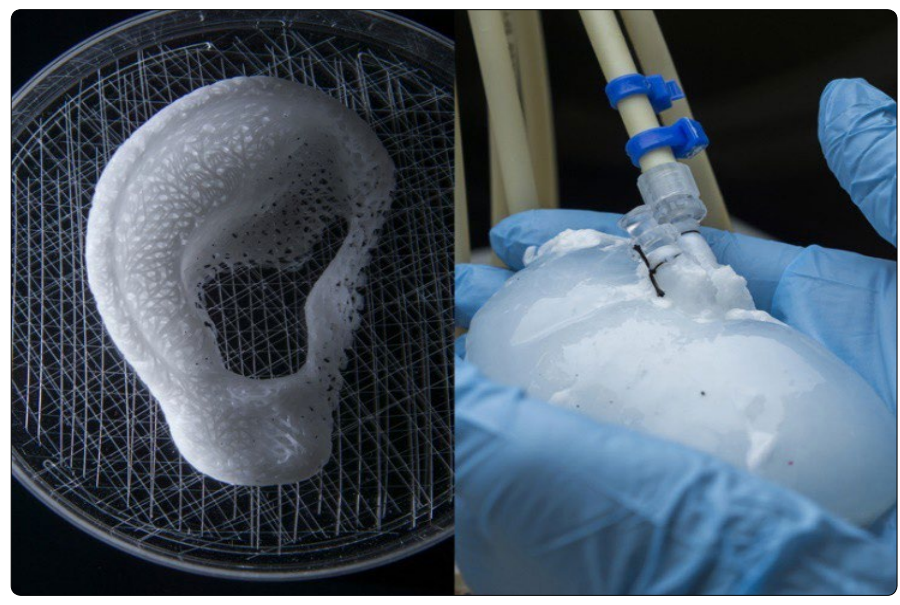

Figure 1. Pictured on the left is a 3D-printed ear scaffold. On the 
right, a kidney stripped of cells intended for scaffold use [17].

Scaffolds are designed to facilitate the attachment, proliferation, and formation of the desired cell type. Alterations to the scaffold architecture or composition may induce formations in/of the seeded stem cells. By the very nature of the scaffold, the scaffold and cells must physically touch and, therefore, interact. So, a bioactive scaffold engages the cells with various physical cues or by stimulating biological signals to influence cell morphology and alignment [5]. For example, cell-adhesive ligands or bioactive cues released by the ECM (such as the RGD sequence on fibronectin) can trigger binding and stimulate specific cellular alignment [5]. Similarly, by mimicking the mechanical properties of the desired tissue, a scaffold will affect the differentiation of stem cells [5]. Tissue engineers may even pre-vascularize the scaffold or seed the cells on a scaffold that already contains blood vessels to promote in growth of a specific tissue [5].

The discovery that the mechanical properties of the scaffold have a huge effect on the stem cells placed on them was an incredibly influential one. But, it was arguably an even greater discovery that the biomechanical environment a tissue experiences naturally improves tissue development and maintenance [6]. It is evident that muscles atrophy when they are not in use for an extended period of time. A reduction in the force experienced by a muscle will lead to a decrease in cell size and number, as well as a decrease in protein synthesis. It was this principle that gave rise to the use of bioreactors in the lab to improve results when directing stem cells differentiation. It is widely accepted that biophysical signals influence cell physiology in vivo; the same principle is applied to engineered tissues in vitro using bioreactors [6].

Bioreactors are in vitro cell culture systems that provide controlled mechanical and/or electrical stimuli to tissueengineered constructs to improve or accelerate the generation of functional tissue [6]. By putting the engineered tissue under biomimetic conditions, it is possible to stimulate ECM synthesis, improve structural organization of the tissue, direct cell differentiation, and enhance the constructed tissue function [6]. To be more specific, bioreactors are designed to expose the developing constructs to convective mixing, perfusion, and overall mechanical conditioning [6]. Additionally, these culture systems control cell distribution on a scaffold and provide efficient mass transfer of nutrients, gases, and regulatory factors to the cells as part of a scaffold-cell construct [6]. With these design specifications come two major considerations necessary to develop or make use of the proper bioreactor.

Mass transfer and transmission of mechanical signals are two requirements for a bioreactor that come with their own considerations [6, 7]. Mass transfer considerations being the considerations that come with maintaining a tissue, whether that tissue is inside a living body or not. First, the bioreactor must be capable of external and internal mass transfer. That is to say that nutrients, oxygen, and bioactive agents must be transported efficiently from the media to the tissue surface and from the tissue surface to individual cells [7]. Likewise, waste products like metabolites and carbon dioxide must be removed from the cell through the tissue matrix to the surface, and then to the media [7]. Both internal and external rates depend largely on the hydrodynamic conditions inside the bioreactor. It is necessary to induce diffusion and convection within the cells by subjecting the bioreactor to media perfusion [7]. It is also necessary to consider how mechanical loads imposed on the scaffold-tissue construct may potentially deform the scaffold, thereby altering the capability of the tissue for mass transfer [7].

It is necessary, of course, to consider mechanical loads because the transmission of mechanical signals is the second consideration when choosing a bioreactor for cells. It is critical to simulate the correct microenvironment based on the cell type that is intended to form $[6,7]$. For example, smooth muscle cells are subjected to and must facilitate the flow of bodily fluids or fluids processed by the body. Smooth muscle cells constantly experience pulsatile flow, and so a bioreactor that contains smooth muscle cells must subject the scaffoldtissue to pulsatile flow $[6,7]$. Other microenvironment specifics include: Skeletal myocytes must be subjected to tensile stress, cardiac myocytes to pulsatile tensile stress [6, 7]. Chondrocytes require compressive stress $[6,7]$. Endothelial cells require pulsatile flow; just as smooth muscle cells do [7].

Essentially, the bioreactor consists of a scaffold-cell construct submitted to specific mechanical stimuli from within a structure or device. Bioreactors can be as simple as spinner flasks that suspend scaffolds in media from within the flask, contain side arms with vented caps that allow for gas exchange, and utilize a magnetic stir bar to generate continuous mass flow. Bioreactors can also be much more complex. To give a more specific example, engineered ligaments must be subjected to dynamic torsion and tension/compression (Fig. 2) [6]. Ligaments are currently a popular tissue request, due largely to the difficulties associated with repairing or replacing the anterior cruciate ligament $(A C L)$. Ligaments like the $A C L$ are oriented axially or wound about a long axis such that during movements they are heavily exposed to axial tensions upwards of $700 \mathrm{~N}$ and torsions of up to $140^{\circ}$ [6]. When fabricating a ligament, advanced bioreactor system must constantly provide these multidimensional mechanical strains without reaching or exceeding the maximum typical value experienced in the body by the ligament.

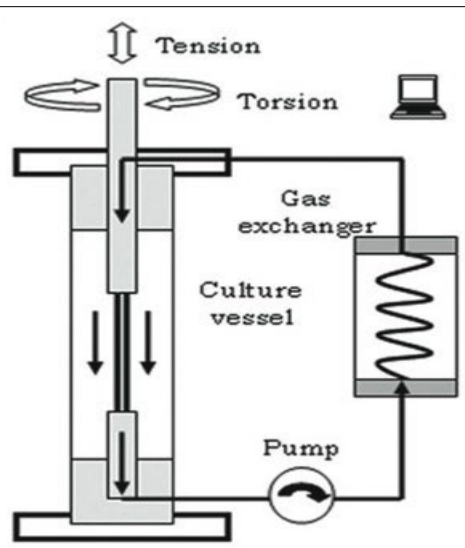

Figure 2. Schematic of a bioreactor intended for ligament production [6]. 
Engineering osteocytes also requires a more refined bioreactor. Bone has a very high compressive modulus that ranges from 10 to $50 \mathrm{MPa}$ [6]. Since bioreactors are intended to simulate the natural environment of the tissue within it, it would follow that a bone bioreactor would impose compressive force. However, tissue engineers found that compressive stimulation from within a bioreactor had little effect on in vitro bone growth and mineralization [6]. This was because the load was experienced almost completely by the scaffold, with almost none of the compressive force being transferred to the stem cells on the scaffold. Further research led tissue engineers to discover that inducing hydrodynamic shear considerably enhances engineered bone growth by perfusion of media that mimics the interstitial flow of canalicular spaces [6]. So, instead, current bone bioreactors direct the flow of media through the scaffold-tissue construct to expose all cells within the construct to shear stress (Fig. 3) [6].
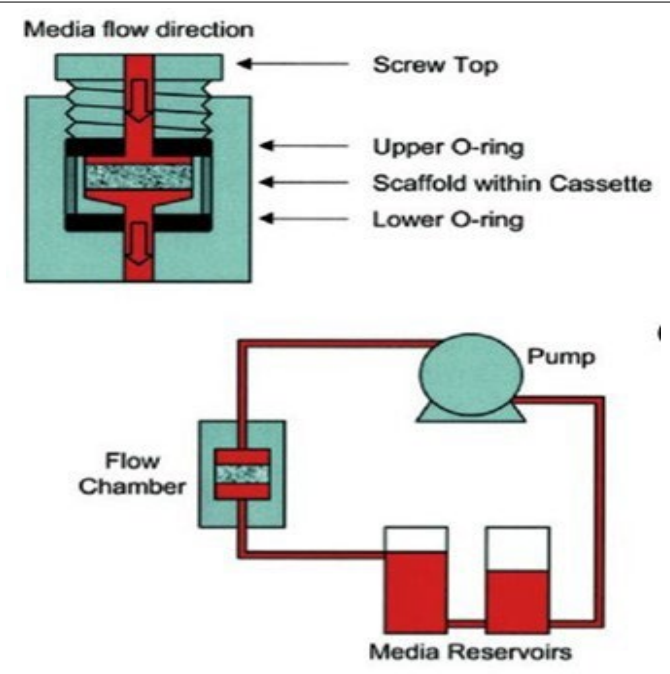

Figure 3. Schematic of a single perfusion bioreactor [6].

Development of these bioreactor systems has ultimately led to the creation of bioreactors for heart valves or major arteries. Aortic valves, aortas, and cardiomyocytes all require extensive pre-conditioning in a bioreactor to ensure that they are robust and fully functioning before implantation. For this reason, these bioreactors will typically require five to seven design components to maximize mass transfer and mechanical loading of the cells [6]. Components may include peristaltic pumping, pressure transducers, fluid capacitance, and electrical stimulation. Electrical stimulation has proven successful with engineered cells, particularly for cardiomyocytes [6]. Within the first three days of development, cells left to incubate can be connected to a cardiac stimulator [6]. Cardiaclike electrical stimuli are applied to induce contractions in the cultured constructs [6].

All of these simulated conditions prepare constructs extensively for eventual implantation. Bioreactor culture systems compared to a static culture have proven to increase the numbers of uniformly distributed cells. Cells from a bioreactor were also larger and greater in number with far greater structural integrity than their statically cultured counterparts. For these reasons, bioreactors have been widely accepted among tissue engineers as a necessary tool to engineer a viable construct for implantation.
All tissue engineers experiment with stem cells, scaffolds, and bioreactors in the hopes that they may eventually create a construct that is ready for clinical trials. The hope, of course, being that positive results will eventually lead to a commercialized product that can better a patient's life. Quite a few examples were found regarding the use of mesenchymal stem cells for alveolar bone tissue engineering for cleft lip and palate patients.

A cleft lip and palate is a congenital defect that forms in the womb. At approximately 5-6 weeks of gestation, the maxillary prominences of the fetus improperly fuse thereby creating a split or opening in the lip and roof of the mouth (Fig. 4) $[8,9]$. An affected child can expect to suffer from speech and hearing disorders, which may, in turn, affect academic capabilities [8]. The conventional correction procedure aims to close the oronasal fistula by restoration of maxillary arch continuity [8]. Bone grafting of the cancellous bone harvested from the patient's anterior iliac crest is the "gold standard" to treat a cleft lip and palate $[8,10]$. However, bone grafting is an invasive procedure that carries a number of potential complications $[8,10]$. In addition to pain, infection, fracture, scarring, chronic pain, paresthesia, misalignment dentition, and midfacial retrusion, the donor site risks postoperative temporary mobility impairment and even donor site morbidity $[8,10]$. For these reasons, treatment options have turned to tissue engineering to avoid autologous bone grafting by instead forming new bone with the patient's stem cells.

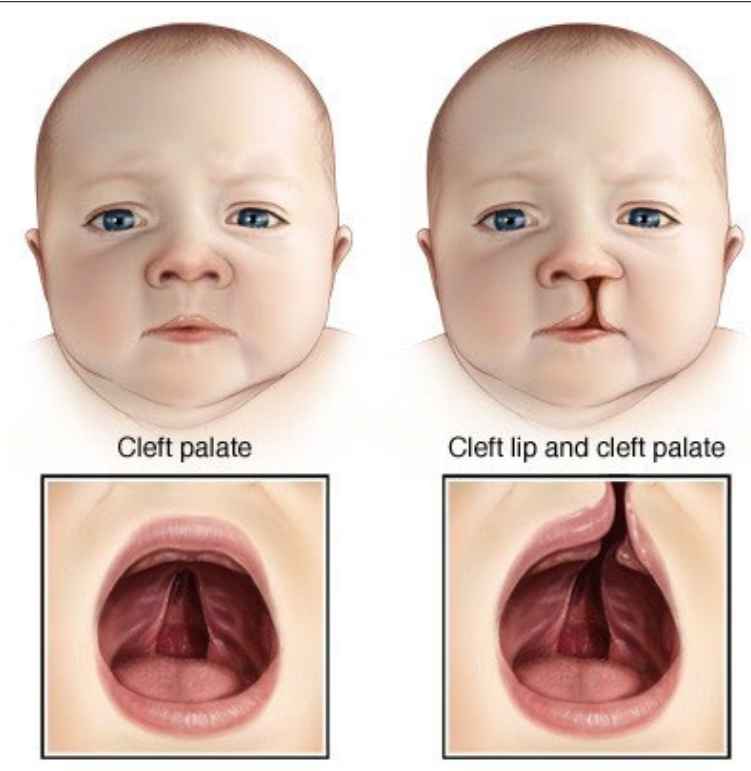

Q MAYO FOUNDATION FOR MEDICAL EDUCATION AND RESEARCH. ALL RIGHTS RESERVED.

Figure 4. On the left is a depiction of an infant with a cleft palate. On the right is a child with a cleft lip and palate [18].

Mesenchymal stem cells (MSCs) are of particular interest primarily because they are capable of differentiating into osteogenic cells $[8,10]$. They are also influenced by paracrine effects, thereby prompting them to release cytokines, produce $\mathrm{ECM}$, and promote angiogenesis; all of which are necessary to establish integration of a scaffold-tissue construct with the host tissue [8]. MSCs are "sufficiently available" in patient bone, cartilage, tendon, muscle, and adipose tissue $[8,10]$. 
These stem cells can be paired with an osteoconductive threedimensional scaffold for best results. Previous studies have observed that using demineralized bone matrix as a scaffold for a bone graft demonstrated no significant difference in the rate of ossification than a traditional autogenous bone graft [10]. Instead, one study investigated whether silicon oxidehydroxyapatite- tricalciumphosphate scaffolds would yield better results in conjunction with MSCs.

The experiment tested whether a significant difference could be identified between control models, grafts with osteogenic differentiated cells, and also grafts with undifferentiated MSCs. All of the tested groups were implanted into a rat model with an artificially created bone defect. After six weeks, the width of the defect had been reduced in the subjects implanted with the tissue-engineered bone grafts [10]. Interestingly enough, results also showed that there were statistical differences between the bone graft groups. Grafts with undifferentiated cells were more efficient with respect to defect reduction than the grafts containing osteogenic cells. These observations were analyzed histologically and also using cone-beam computed tomography; however, the observations were also evident histomorphologically [10].

Clinical trials throughout the years have yielded promising initial results. The first clinical use of stem cells for bone tissue engineering was performed on a patient with an alveolar cleft in 2006 [8]. The patient had undergone surgical correction eight years prior with little success. Nine months following the procedure, $79.1 \%$ of the bone had regenerated [8]. In 2009, another patient that had little luck with his two previous surgeries attempted a tissue- engineered transplantation. A follow-up conducted 18 months later showed "complete defect closure" [8]. An ongoing clinical trial conducted by the Sirio-Libanes Hospital has reported positive findings for a preliminary study $[8,9]$. The clinical trial observed 5 patients with alveolar clefts and the effect of implanting autogenous MSCs derived from deciduous dental pulp with a scaffold [8, 9]. 6 months after surgery, all patients had experienced alveolar bone union with a mean of $89.5 \%$ bone regeneration. The quality of bone regeneration has yet to be determined.

Much of the success of these trials has been attributed to the careful selection of stem cell source, scaffold, and growth factors [8]. To start, the inclusion of angiogenic factors had an incredibly positive impact on trials. A major obstacle for longterm bone stability and functionality is effective vascularization; thus, it was hypothesized that the promotion of vascularization would accelerate tissue regeneration $[4,8]$. Inclusion of tissueengineered components with pro-angiogenic properties promoted the formation of a vascular network that then promoted enhanced bone formation [8]. Placing premature scaffolds into a bioreactor enhanced vascular promotion even further. Selection of a scaffold whose composition and overall porosity mimic the microenvironment of bone also positively influenced MSC regeneration [8]. Inclusion of these factors was coupled with the use of MSCs, which have demonstrated success differentiating into the osteogenic lineage in addition to high integration with scaffold technology. That does not begin to cover, however, the difference MSCs from various locations have made on trials, scaffold materials, methods of improving the osseoconductivity between the cells and scaffold, etc.

Obviously, there is considerable variability associated with constructing a tissue or organ for a patient. Engineers must discover or create the ideal combination of stem cells and scaffolding for patient use. First of all, stem cell type (autologous vs allogeneic) and donor site must be determined. Then, researchers face the arduous process of engineering a scaffold that will augment tissue integration with the body after inducing differentiation of the desired tissue type. When products are finally sufficiently developed, engineers may find that a construct suitable in vitro is not equally suitable in vivo. To recreate the biological complexity of an organ in-lab, tissue engineers have created biological micro-electro-mechanical systems (BioMEMS). BioMEMS allow researchers to experiment with a precise organ system on a microscale (Fig. 5), commonly known as an organ-on-a-chip (OOC) [11, 12].

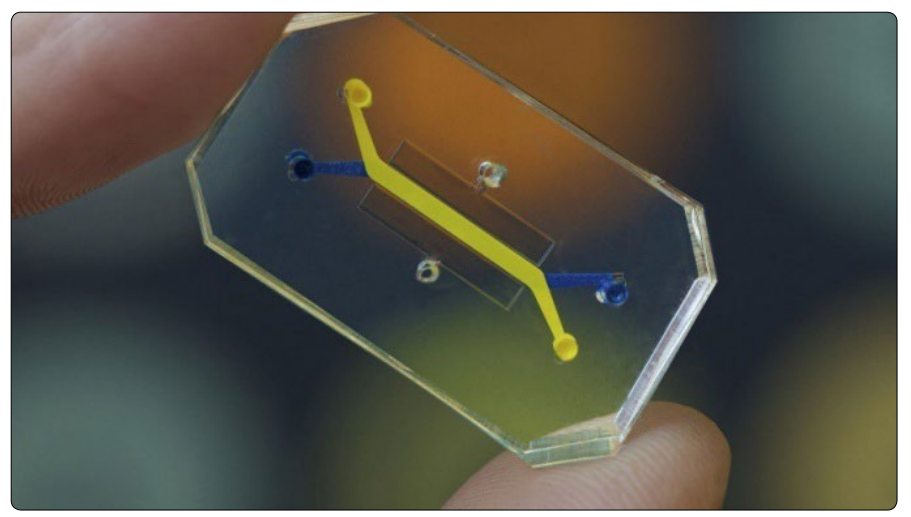

Figure 5. The airway-on-a-chip, a modified design of the lung-ona-chip. Both developed by the Harvard Wyss Institute [13].

An OOC is typically used for point-of-care diagnostics, such as toxicity screenings or drug discoveries [11,12]. However, by mimicking or recapitulating human-specific pathophysiology, researchers can use BioMEMS to engineer tissue replacements with faster results. This is especially possible, as OOCs can be made as simple or complex as researchers require. Meaning that researchers can make developments piece by piece to directly account for individual processes that might otherwise be masked in a host and potentially interconnect multiple OOC systems into a network to emulate whole inter-organ relationships [11,12]. BioMEMS also offer a few additional applications to further tissue engineering, including the formation of vascular networks and engineering vascular beds for whole organs and micro fluidic bioreactors for screening stem cells and the establishment of patterned tissue interfaces [11].

As previously stated, creating an intrinsic vascular supply poses a significant challenge. A vascular network is necessary to maintain a viable tissue and crucial to avoid developing necrotic regions in an organ. Due to this fact, researchers are pursuing microfabrication techniques to replicate highly efficient mass transfer. The $\mathrm{OOC}$ platform is a construct capable of forming vascular networks on a micron scale (i.e. a capillary-like network) [11]. In fact, BioMEMS are particularly 
well suited for constructing scaffolds and bioreactors that achieve a highly vascularized structure in the target organ [11]. Early work has discovered that introducing growth factors to poly (lactic co-glycolic acid) (PLGA) scaffolds in a stepwise function results in the development of a stable micro vascular network [11]. Pairing this discovery with human embryonic stem cells generates a well-vascularized skeletal muscle tissue [11]. Additionally, experiments have been made with nanostructured bioresorbable elastomers as scaffolding to develop a liver replacement that enhances primary hepatocyte function [11].

Regarding, stem cell screening, it is an accepted truth that stem cells respond to "the entire context of their environment" both in vitro and in vivo [11]. For this reason, utilizing culture systems to replicate a cell niche could possibly unlock the full potential of stem cells for tissue engineering [11]. The current theory stands that using microfluidic platforms to precisely manipulate the microenvironment is key to study cellular responses in real time [11]. Microfluidic techniques have already led to the widespread redesign of scaffolding as it was previously understood to include micropatterning of cells and different biomaterials [11]. During the course of these studies, microfluidic systems have also revealed factors involved in regulation of stem cell regulation [11]. Instead of constantly changing media, as is the case in a well plate, utilizing microarray bioreactors with medium perfusion creates a tightly controlled culture environment ideal for stem cell screening [11].

A recent set of studies used this technique in conjunction with human embryonic stem cells and found a correlation between cell differentiation and hydrodynamic shear [11]. A correlation was also found between cell differentiation and oxygen/growth factor transport rates [11]. The implications of these studies will be instrumental to perfecting a tissueengineered construct. By tightly controlling the fluid dynamics within the culture system, it is possible to engineer complex tissue structures from undifferentiated stem cells [11]. Introducing controlled concentration gradients to cell culture space has also revealed that the delivery of the expression modulator doxycycline (Dox) can effectively pattern the expression of the BMP-2 gene and thereby modulate osteogenic differentiation [11]. In short, BioMEMS have helped further understanding of optimal scaffold-tissue development, and also how to more reliably direct differentiation of stem cells.

The preliminary focus thus far has been how tissue engineers currently attempt to construct tissue-engineered products with various human stem cells for human use. However, tissue engineers don't necessarily focus their efforts on constructing new tissues or organs that must be implanted into a patient. Considerable research has also been made into adult stem cells and their potential for directed healing from within a host body. Adult stem cells are undifferentiated cells found among differentiated cells whose role is to maintain and repair tissues [16]. However, these stem cells are not restricted to only the tissue they are originally found in. Many adult stem cells exhibit high plasticity and may differentiate into a number of cell types [14]. For example, bone marrow stem cells may differentiate into certain brain cells, liver cells, and even skeletal and cardiac muscle cells [14]. It is for this reason that current research is also aimed at determining the mechanisms that underlie adult stem cell plasticity [14]. If such mechanisms can be identified and controlled, it might be possible to induce or direct stem cells in the adult body to repair diseased tissues, even if they are of a different tissue type.

This type of research toes the line between regenerative medicine and tissue engineering. For now, most approaches require expanding autologous stem cell populations, providing the culture with additional bioactive factors and sometimes a scaffold, then returning them to their original tissue [15]. However, ex vivo expansion is incredibly difficult to maintain at this point in time. The future of ex vivo expansion lies, again, with understanding cell behavior, including cell proliferation and understanding the contributions of the original tissue type. For example, tissues expanded for reconstruction tend to have much higher rates of success if the original tissue experiences high turnover rates in the body (e.g. blood, epithelium) [15]. Recent studies have also shown, too, that it is possible to reprogram certain adult cell types in vivo via genetic modification [16]. This strategy may possibly yield a way to create cell types that had previously been lost or damaged by disease [16].

As far as current knowledge regarding adult stems cells, there are still key questions surrounding their use. Researchers are not certain that adult stem cells exhibit plasticity and transdifferentiate normally, or if transdifferentiation is a property that is only observed when cells are manipulated experimentally [16]. There are also the questions of what factors stimulate stem cells to relocate to sites of injury, disease, or damage and whether or not it is possible to enhance that homing process [16]. These questions obviously affect the ability of tissue engineers to direct stem cells to a site and initiate differentiation as an option in regenerative medicine.

\section{Conclusion}

Because tissue engineering and stem cell research have emerged so recently, much is still unknown about stem cell behavior and how to engineer stem cells exactly to a desired specification. Researchers discovered scaffolding had the potential to hugely influence stem cell differentiation. Soon thereafter, it was discovered that placing a scaffold-tissue construct in a bioreactor with specific mechanical loading could enhance the ability of the cells to form a tissue. Bioreactors were shown to improve vascularization and desired physical properties of the engineered tissue. However, despite the advances made in the field, tissue engineering is still restricted by experimentation.

Tissue-engineered constructs must ultimately be inserted into a body to see if the construct can achieve full host integration and its desired effect (i.e. bone regeneration, indefinite aortic function). Sadly, most tissue-engineered 
products will not progress to the clinical trial phase. Rigorous regulations and an expensive process require approximately a decade's worth of effort to gain approval to implant a product into a human being. Even if surgical results are initially promising, researchers can't know if the construct is successful without further analysis months, oftentimes years, postoperation. Tissue engineers have developed organs-on-a-chip, or BioMEMS, to combat the difficulties presented by this lengthy process. Engineers are now able to observe microenvironments seeded with human cells, with or without an included engineered tissue, in real time. The use of BioMEMS continues to accelerate the field of tissue engineering.

Within the field of tissue engineering, research is also being made to direct host stem cells from inside the patient's body. Engineers are researching methods of relocating the patient's own stem cells to a diseased or damaged site and differentiate accordingly. Theoretically, a scaffold-tissue construct would not even be necessary if engineers could boost the patient's natural healing process using their own stem cells. More and more discoveries are being made that significantly improve the field and its products, as we know it. As the future of tissue engineering brightens, so does the future of medicine.

\section{References}

1. Eberli $D$, Atala A. Tissue engineering using adult stem cells. Methods Enzymol. 2006; 420: 287-302. doi: 10.1016/s0076-6879(06)20013-2

2. Organ Donor Statistics. U.S. Department of Health \& Human Services. Aug 2017.

3. A Step-by-Step Guide to the MACI Procedure. MACI. Verticel Corporation. 2017.

4. Howard D, Buttery LD, Shakeseff KM, Roberts SJ. Tissue engineering: strategies, stem cells and scaffolds. J. Anat. 2008; 213(1): 66-72. doi: 10.1111/j.1469-7580.2008.00878.x

5. Chan BP, Leong KW. Scaffolding in Tissue Engineering: General Approaches and Tissue-specific Considerations. Eur Spine J. 2008; 17(4): 467-79. doi: 10.1007/s00586-008-0745-3
6. Lanza R, Langer R, Vacanti JP. Principles of Tissue Engineering. London: Academic/Elsevier, 2015.

7. Salehi-NN, Amoabediny G, Pouran B, Tabesh H, Shokrgozar MA, et al. Engineering Parameters in Bioreactor's Design: A Critical Aspect in Tissue Engineering. BioMed Research International. 2013. doi: 10.1155/2013/762132

8. Gladysz D, Kamil KH. Stem Cell Regenerative Therapy in Alveolar Cleft Reconstruction. Archives of Oral Biology. 2015; 60(10): 1517-532. doi: 10.1016/j.archoralbio.2015.07.003

9. Bueno DF. Clinical Trial: Use of Mesenchymal Stem Cells for Alveolar Bone Tissue Engineering for Cleft Lip and Palate Patients. Hospital Infantil Universitario Niño Jesús, Madrid, Spain. 2015.

10. Korn P, Schulz MC, Range U, Lauer G, Pradel W. Efficacy of Tissueengineered Bone Grafts Containing Mesenchymal Stromal Cells for Cleft Alveolar Osteoplasty in a Rat Model. Journal of Cranio-Maxillofacial Surgery. 2014; 42(7): 1277-285. doi: 10.1016/j.jcms.2014.03.010

11. Borenstein JT, Gordana GV. Engineering tissue with BioMEMS. IEEE Pulse. 2011; 2(6): 28-34. doi: 10.1109/MPUL.2011.942764

12. Cho $\mathrm{S}$, Yoon JY. Organ-on-a-Chip for Assessing Environmental Toxicants. Current Opinion in Biotechnology. 2017; 45: 34-42. doi: 10.1016/j. copbio.2016.11.019

13. Wyss Institute. Human organs on a chip.

14. Catacchio I, Berardi S, Reale A, et al. Evidence for Bone Marrow Adult Stem Cell Plasticity: Properties, Molecular Mechanisms, Negative Aspects, and Clinical Applications of Hematopoietic and Mesenchymal Stem Cells Transdifferentiation, Stem Cells International.2013. doi:10.1166/2013/689139

15. Lanza R, Gearhart J. Essentials of Stem Cell Biology. Amsterdam: Elsevier Academic. 2009

16. National Institutes of Health. Stem Cell Basics IV. 2016

17. Steve V. Engineering New Organs Using Our Own Living Cells. Discover. 2015.

18. Cleft Palate, Mayo Foundation for Medical Education and Research. Mayo Clinic. 2017.

19. Al-Ayoubi AM, Rehmani SS, Sinclair CF, Lebovics RS, Bhora FY Reconstruction of Anterior Tracheal Defects Using a Bioengineered Graft in a Porcine Model. The Annals of Thoracic Surgery. 2017; 103(2): 381-389. doi: 10.1016/j.althoracsur.2016.10.034 